\title{
A Simulation Study for the attenuation correction of Respiratory Motion with the PET/CT Gating data
}

\author{
Lei $\mathrm{Fu}^{1, \mathrm{a}}$, Yu Hong ${ }^{2, \mathrm{~b}}$, Yunhai $\mathrm{Ji}^{3, \mathrm{c}}$ and Jianfeng $\mathrm{He}^{4, \mathrm{~d}}$ \\ ${ }^{1}$ Institute of Biomedical Engineering of college of Information Engineering and Automation, Kunming \\ University of Science and Technology, Yunnan 650500, China \\ ${ }^{2}$ Kunming Hospital affiliated to General Hospital of the Chinese People's Liberation Army, Kunming , \\ Yunnan 650500, China \\ ${ }^{3}$ The first people's Hospital of Yunnan Province, Kunming , Yunnan 650500, China \\ ${ }^{4}$ Institute of Biomedical Engineering of college of Information Engineering and Automation, Kunming \\ University of Science and Technology, Yunnan 650500, China \\ A782285411@qq.com, bjfenghe@qq.com
}

Keywords: PET/CT; Gating; Respiratory Motion; Attenuation Correction

Abstract. Objective To investigate the effect of attenuation correction on the gating data based on the true coincidences event of detector ring with the application of PET/CT.Methods Use the software GATE that can simulate the imaging of PET/CT to do the pixel phantom simulation in order to simulate the respiratory motion of the lung in the process of PET/CT scanning. Then, do the gating process and the attenuation correction with the simulation data of GATE.Results The quality of gating images with attenuation correction is better than none attenuated images.Conclusion The quality of gating images with attenuation correction can further improve the quality of images and the accuracy of diagnosis.

\section{Introduction}

The quality of PET/CT image is influenced by a lot of factors in the process of PET/CT scanning imaging. Among many factors, the attenuation effect is an important factor to affect the image quality. The study indicates that the attenuation effect not only can cause the lost of counting and the quantitative inaccuracy,but also can cause the image nonuniformity and distortion phenomenon,therefore,we need to do the attenuation correction for the PET/CT image.Before the combination of PET and CT, the conventional method is transmission scan.This is a way that use the arc penetration source to detect the objects in the transmission field of vision in order to obtain the data of each direction projection line and calculate the tissue attenuation coefficient for each position. The process of obtaining the attenuation correction coefficient is relatively simple when use the transmission scan based on coincidence events to do the attenuation correction. The advantage of this method is that there is no need to convert the photon energy.However,it limits the count rate that the coincidence events could be achieved.In addition, the time required for a transmission scan is too long, and a FOV scan takes 10 to 15 minutes[1].After the combination of PET and CT, the CT image is used to do the attenuation correction for PET.This is a way that the attenuation factor of CT image is converted into PET attenuation factor accurately.The structure of CT image has high resolution, which can reduce the influence of tissue attenuation on image quality and improve the accuracy of the image.As the photon energy in PET and CT is not the same,the CT transmission scanning will not interfere with the PET scanning data, and the scanning speed of CT is fast so that can shorten the scan time[2].

In this paper,we use the GATE simulation platform and do the attenuation correction for the gating image on the basis of gating correction of respiratory motion.NCAT is used for the simulation experiment.the results show that the method can reduce the impact of tissue attenuation in the image, thereby further improving the quality of the image. 


\section{Materials and Methods}

\section{Materials}

The clinical scanning simulation equipment of respiratory motion in the PET/CT is GE Discovery LS.The main physical parameters of the PET/CT scanning device are as follows:

Scan mode: 3D scan FOV:152mm Detector ring diameter: $92.7 \mathrm{~cm}$

The scanner has 18 detection rings, each of which has 672 detectors.

Scintillation crystal detector materials: bismuth germanium bismuth (BGO)

BGO size is 4 Trans-axial mm, 8 Axial mm,30 Radial mm

Data analysis and processing software: ROOT

Image analysis and processing software: Matlab

\section{Methods}

By using the Gate simulation software, the simulation phantom is put into the PET scanning equipment.The principle of the gating method based on the true coincidence event of the detector rings is: When the phantom is moving in the PET scanne,the position of the detector in the detector is changed.In the same time interval, the distribution of the number of photons in the PET detector rings will be changed accordingly.The phantom in the PET detector moves from right to left,The distance of each moving is the same as the width of a detecting ring. The position of the peak of the true photon number distribution of the detected ring is also moving from right to left.And each moving distance is the width of a detecting ring[3].

After do the Gating correction for the image,and then the attenuation correction is carried out. As is shown in Fig. 1, the principle of attenuation correction is:

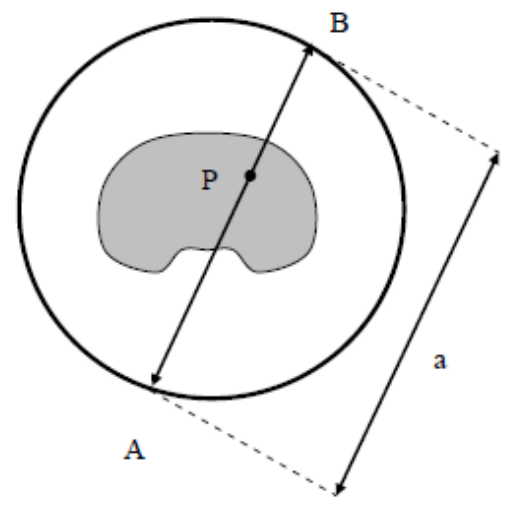

Fig. 1

Assuming that the point $\mathrm{P}$ is the annihilation point, it is known that the distance between $\mathrm{A}$ and $\mathrm{B}$ is $\mathrm{a}$, and two photons are detected by the detector A and the detector B respectively. the probability of these two photons be attenuated is :

$P_{d}=P_{1} P_{2}=e^{-\mu P A} e^{-\mu P B}=e^{-\mu A B}=e^{-\int_{0}^{a} \mu(x) d x}$

One of the photons walks through PA, and the attenuation probability of it on PA is:

$P_{1}=e^{-\mu P A}=e^{-\int_{0}^{P} \mu(x) d x}$

The other of the photons walks through $\mathrm{PB}$, and the attenuation probability of it on $\mathrm{PB}$ is:

$P_{2}=e^{-\mu P B}=e^{-\int_{P}^{a} \mu(x) d x}$

Therefore, the attenuation correction factor of $\mathrm{AB}$ is:

$A C F=e^{-\int_{0}^{a} \mu(x) d x}$

From the above, we can complete the attenuation correction by multiplying the attenuation correction factor and the original sinusoidal data together to obtain a more accurate diagnostic image[4][5]. 


\section{Implementation steps}

The original data of PET is divided into $\mathrm{N}$ frame by fixed time interval.Then select one frame as the reference frame (optional), and compare the absolute error between the other frames and the reference frame,the frames that is approximate can be used as the new PET phantom data to get the new gating data.Then the output of gating data is introduced into the MATLAB for attenuation correction.Firstly the phantom of original CT image is read into the MATLAB or other software to obtain the attenuation correction coefficient.Then the $\mathrm{CT}$ attenuation correction coefficient is converted to the attenuation coefficient of PET energy, and the attenuation correction factor ACF is obtained[6].Then multiply it by the gating data of PET phantom,we can get a new PET data.At last, the MLEM algorithm is used to reconstruct the image of PET. The specific steps are as follows:

(1)The simulation software GATE is used to get the original data of PET

(2)The data is divided into $\mathrm{N}$ frames with a fixed time interval.

(3)In each PET data,count the true photon number of each frame of every detection loop

(4)Choose one frame as reference frame (can be arbitrarily selected), respectively, compared with other frames, then calculate the absolute error of the true photon number of each frame of every detection loop.

(5)Compare all of the absolute error values, select the similar absolute error value of the frame data is as the new PET scan data (gating data).

(6)Import the gating data to MATLAB, the correspond NCAT image will be transformed into PET energy attenuation coefficient in the MATLAB,then calculate the attenuation correction factor(ACF) on each LOR according to the formula 1, and multipy the gating data by the ACF, we can get the new PET data at last(the gating data after attenuation correction)

(7)Using MLEM iterative algorithm to reconstruct the image with the new scan data.

\section{Simulation experiment}

The main software used in this paper is NCAT, GATE, ROOT and MATLAB.NCAT is generated based on the real patient datas, and can accurately simulate the real environment of human body[7].GATE is an advanced opensource software developed by the international OpenGATE collaboration and dedicated to numerical simulations in medical imaging and radiotherapy[8][9]. The simulation experiments uses the pixel phantom (NCAT) and obtains the original simulation data for gating process through ROOT, then achieves the attenuation correction and image reconstruction for the gating data through MATLAB.

\section{Pixel phantom simulation experiment}

In order to verify the method proposed in this paper can effectively improve the gating data, this experiment uses NCAT to simulate the lung respiratory movement in the real life. The NCAT body model is introduced into the GATE simulation platform to simulate,the specific parameters are as follows:

Lung pixel size: $128 * 128 * 31$; Each pixel represents the actual size: $3.125 * 3.125 * 4.25 \mathrm{~mm}$;

Radioactivity in the lungs: $18 \mathrm{~F}-\mathrm{FDG}$ 5000bq;Tumor activity: 30000bq;

Radioactivity in the liver was 40000bq;

The original PET data is divided into 100 frames with $200 \mathrm{~ms}$; The respiratory cycle is $5 \mathrm{~S}$, the simulation time is 4 cycles(20s);

\section{Simulation results and image evaluation}

The analysis and processing of raw data and image reconstruction

The key technology lies in the basic research for the attenuation correction of Respiratory Motion with the PET/CT Gating data is the analysis and processing of the PET raw data and the calculation of the attenuation correction factor. Then use ROOT for image processing, and use MATLAB to do the attenuation correction, finally you can get a PET image with gating correction and attenuation correction.In addition, as is shown in Fig. 2 and Fig. 3, the simulation experiment of this paper increases the static lung image and the motion blurred image of the lung in order to make an objective 
comparison and analysis.Fig. 4 is the gating image without attenuation correction.Fig. 5 represents the gating image after attenuation correction, the gating image without attenuation correction means to deal the original data of motion blurred lung with the gating correction based on the true coincidence events of detector rings and without the attenuation correction. The gating image after attenuation correction means to deal the original data of motion blurred lung with the gating correction based on the true coincidence events of detector rings and the attenuation correction.

We can know from the the Fig. 2,Fig. 3 Fig. 4 and Fig. 5 that The shape and the size of the lungs in the Fig. 4 is close to those of the static lung image.However,The shape and the size of the lungs in the Fig. 5 comes closer when compared with Figure 4.

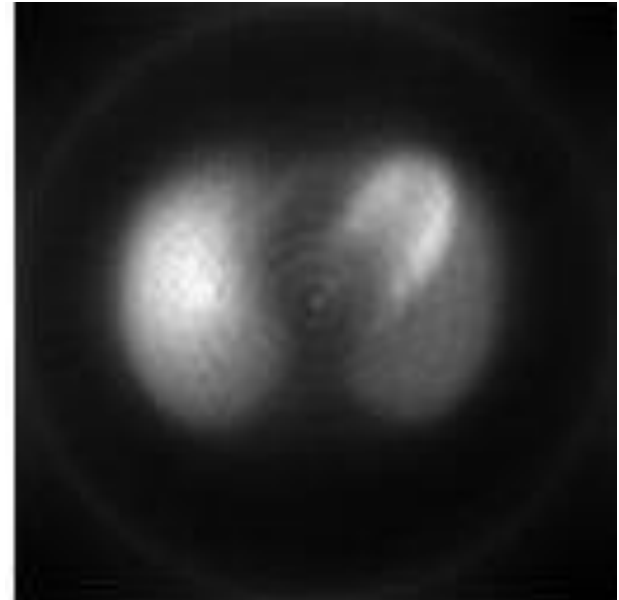

Fig. 2 the motion blurred lung

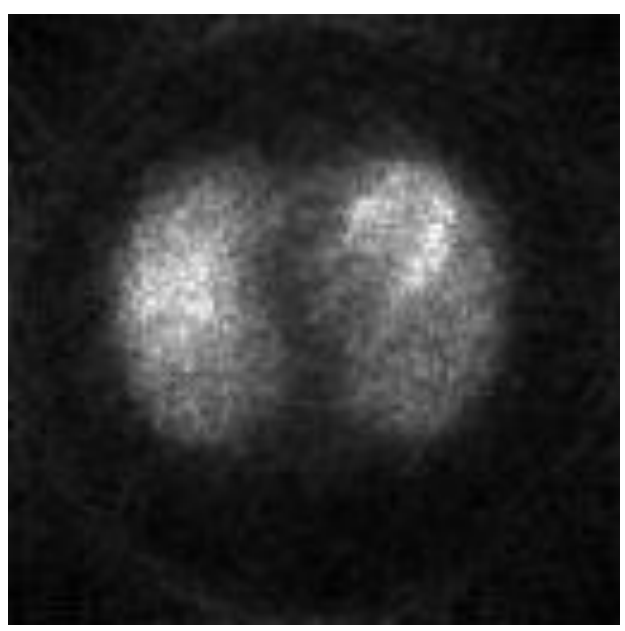

Fig. 4 the gating image without attenuation correction

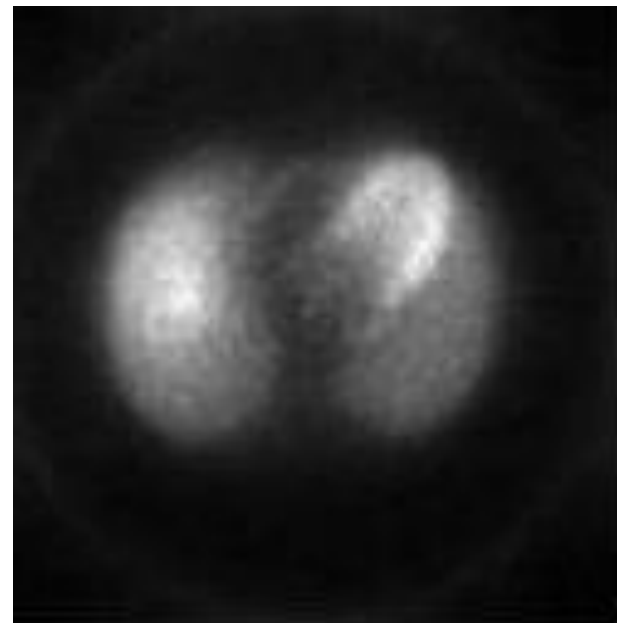

Fig. 3 the static lung

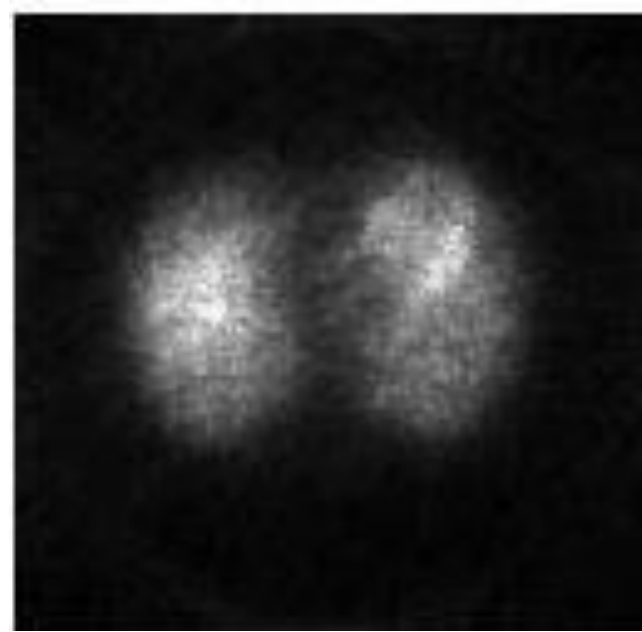

Fig. 5 the gating image after attenuation correction

Through the analysis of the experimental results above, compared with gating correction for the respiratory motion based on the true coincidence events of detector rings, the gating image with attenuation correction, which further improves the accuracy of the image. However, only through the qualitative analysis can not fully prove this point. So this paper will use the results of quality evaluation[10] to analyze the experimental results.

Result evaluation

As shown in Table 1,use the motion blurred image,the gating image without attenuation correction, and the gating image after attenuation correction to compare with the static image through the MATLAB program.MSE is the average value of the error mean square of the gray value of corresponding pixel of the static image and the motion blurred image(or corrected image).The smaller the MSE value is, the smaller difference between them are.PSNR is the pair value of the error mean square of static image and motion blurred image(or corrected image) relative to the maximum value of the pixel.The larger value of PSNR is, the less distortion of motion blurred image or corrected image is,the more realistic the image is.AG is used to measure the clarity of the image. The higher the 
AG value is, the clearer the image is. Therefore,we can conclude that the MSE of the gating image after attenuation correction is smaller than that of the motion blurred image and the gating image without attenuation correction. The evaluation values of PSNR and AG in the gating image after attenuation correction is larger than those of the motion blurred image and the gating image without attenuation correction, which means that the gating image after attenuation correction is more close to the static image than the motion blurred image and the gating image without attenuation correction.

Table 1 image quality evaluation of NCAT simulation results

\begin{tabular}{llll}
\hline & MSE & PSNR & AG \\
\hline motion & 0.00018942 & 85.3564 & 0.0011755 \\
none attenuation & 0.0001611 & 86.3871 & 0.0012882 \\
after attenuation & 0.00012418 & 87.1904 & 0.0014949
\end{tabular}

The results of quantitative analysis showed that compared with the motion blurred image and the gating image without attenuation correction, the gating image after attenuation correction is more close to the static image. The gating image after attenuation correction can further effectively solve the respiratory motion effects on PET/CT image, improve the image clarity, shape and size of the lungs and make the outline of lung close to the stationary state.

\section{Conclusions}

In this paper,we verify the effect of the basic research for the attenuation correction of respiratory motion with the PET/CT gating data on the PET/CT respiratory movement.The results prove that compared with the motion blurred image and the gating image without attenuation correction,the gating image after attenuation correction can improve the image clarity and accuracy and accurately measure the true size and position of the phantom, the image quality has been further improved.

\section{References}

[1] Xueli Li and Yong Zhao: PET attenuation correction based on CT images, edtied by CT and 3D imaging academic annual conference proceedings, China(2004).

[2] Qian Zhang, in: The study of attenuation correction and segmentation methods in the process of lung imaging, edtied by Kunming University of Science and Technology Publications,China(2013).

[3] Rui Cui , Jianfeng $\mathrm{He}$ and Zeng $\mathrm{Fu}$ et al: The study on the respiratory motion gating method based on the detector ring true coincidence event distribution, edtied by volume 3 of Journal of Biomedical Engineering, China(2015), p. 281-290.

[4] Yingmao Chen and Jiahe Tian: The attenuation correction techniques for PET imaging, edtied by volume 6 of Chinese Journal of Nuclear Medicine (2002), chapter, 10,p. 382-384.

[5] Zaidi H and Hasegawa B: Determination of the attenuation map in emission tomography, edtied by volume 2 of Chinese Journal of Nuclear Medicine(2003), chapter, 22,p. 291-315.

[6] McQuaid S J and Hutton B F: Sources of attenuation-correction artefacts in cardiac PET/CT and $S P E C T / C T$, edtied by volume 6 of European journal of nuclear medicine and molecular imaging(2008), chapter, 35,p. 1117-1123.

[7] He JF and O'Keefe GJ, et al:Application of GATE and NCAT to respiratory motion simulation in alle gro PET, edtied by IEEE Nuclear Science Symposium Conference Record. San Diego (2006),p. 2589-2593.

[8] Lamare F, Turzo A and Bizais Y, et al: Validation of a Monte Carlo simulation of the philips allegro/GEMINI PET systems using GATE,edtied by volume 4 of Phys Med Biol(2006), p. 943-962. 
[9] Bert J, Perez-Ponce H and El Bitar Z et al: Geant4-based Monte Carlo simulations on GPU for medical applications Phys, edtied by volume 16 of Med Biol(2013),chapter, 58,p. 5593-5611.

[10] Cerciello, T, Bifulco, $\mathrm{P}$ and Cesarelli, M,et al: A comparison of denoising methods for X-ray fluoroscopic images, edtied by volume 6 of Biomedical Signal Processing and control(2012), chapter, 7,p. 550-559. 